\title{
A survey of submesoscale currents
}

\author{
James C. McWilliams* (1)
}

\begin{abstract}
Submesoscale currents are pervasive throughout the ocean. They have intermediate space and time scales-neither mesoscale nor microscale - that have made them elusive for measurements and modeling until recently. In this brief article, a survey is presented of their primary characteristics and interpretive explanations, intended for a broad audience of physical and biogeochemical oceanographers. Besides their identifying scales, submesoscale currents are distinctive in their flow patterns, their essential dynamical processes, and their consequences for transport, mixing, and dissipation in the general circulation. There are two primary submesoscale populations, a frontal one in the nearsurface layer with its typically reduced stratification, and another vortical one, generated in topographic wakes, that (sparsely) fills the oceanic interior.
\end{abstract}

Keywords: Submesoscale currents, Density fronts and filaments, Coherent vortices, Topographic waves

\section{Introduction}

The ocean is highly variable: just ask any sailor or seagoing experimentalist. Among its modes of variability is a class of phenomena that have come to be called submesoscale currents (abbreviated here as SMCs). The SMC name arose in relation to the widely familiar mesoscale eddies that contain the greatest fraction of kinetic energy in the ocean; i.e., SMCs are the next size class down from the eddies, with typical horizontal lengths of tens to ten thousands of meters, vertical heights of ten to hundreds of meters, and evolutionary time scales of hours to days. ${ }^{1}$ The name is also apt in the sense that the primary source for SMC energy comes from mesoscale eddies by a downscale transfer. The principal SMC generation mechanisms are (1) extraction of available potential energy (due to horizontal buoyancy gradients) in the weakly stratified surface layer either through baroclinic instability or frontogenesis, and (2) topographic-drag vorticity generation in flows along a sloping bottom, followed by boundary current separation and wake instability. These phenomena partly manifest a loss of hydrostatic, geostrophic momentum balance, and they exhibit a turbulent energy cascade forward toward even smaller scales. Thus, SMC dynamics typically go beyond quasigeostrophy, which is the generally

\footnotetext{
*Correspondence: jcm@atmos.ucla.edu

Department of Atmospheric and Oceanic Sciences, UCLA, 405 Hilgard
}

Ave., Los Angeles, CA 90095-1565, USA successful theoretical framework for mesoscale eddies, while still being strongly influenced by Earth's rotation and the generally stable density stratification in the ocean. That is, the Rossby and Froude numbers:

$$
R o=\frac{V}{f \ell} \quad \text { and } \quad \mathrm{Fr}=\frac{\mathrm{V}}{\mathrm{Nh}}
$$

are typically order-one parameters, rather than their usually small values for mesoscale eddies ( $V$ is a characteristic horizontal velocity scale, $\ell$ and $h$ horizontal and vertical length scales, $f$ the Coriolis frequency, and $N$ the Brunt-Vaisala or stratification frequency.) Thus, a Rossby number Ro measures the relative magnitude of momentum advection relative to the Coriolis force, and a Froude number $\mathrm{Fr}$ measures the ratio of an advecting velocity to the phase speed of an internal gravity wave. Small values for these numbers indicate the dynamical importance of rotation and stratification. Nevertheless, the evolution of SMCs is primarily through advection, which distinguishes it from the inter-gravity waves that can occupy similar scale ranges in the ocean; their mutual influence is a topic of current research, but, in some first approximation, their interactions are weak.

The science of SMCs has blossomed in recent years. The delay, compared to other more familiar types of

\footnotetext{
${ }^{1}$ However, Submesoscale Coherent Vortices (SCVs), once formed and freely moving within the interior ocean, can have survival lifetimes of years (McWilliams 1985).
} 


\section{The Flow of Energy and Information in the Oceanic General Circulation}

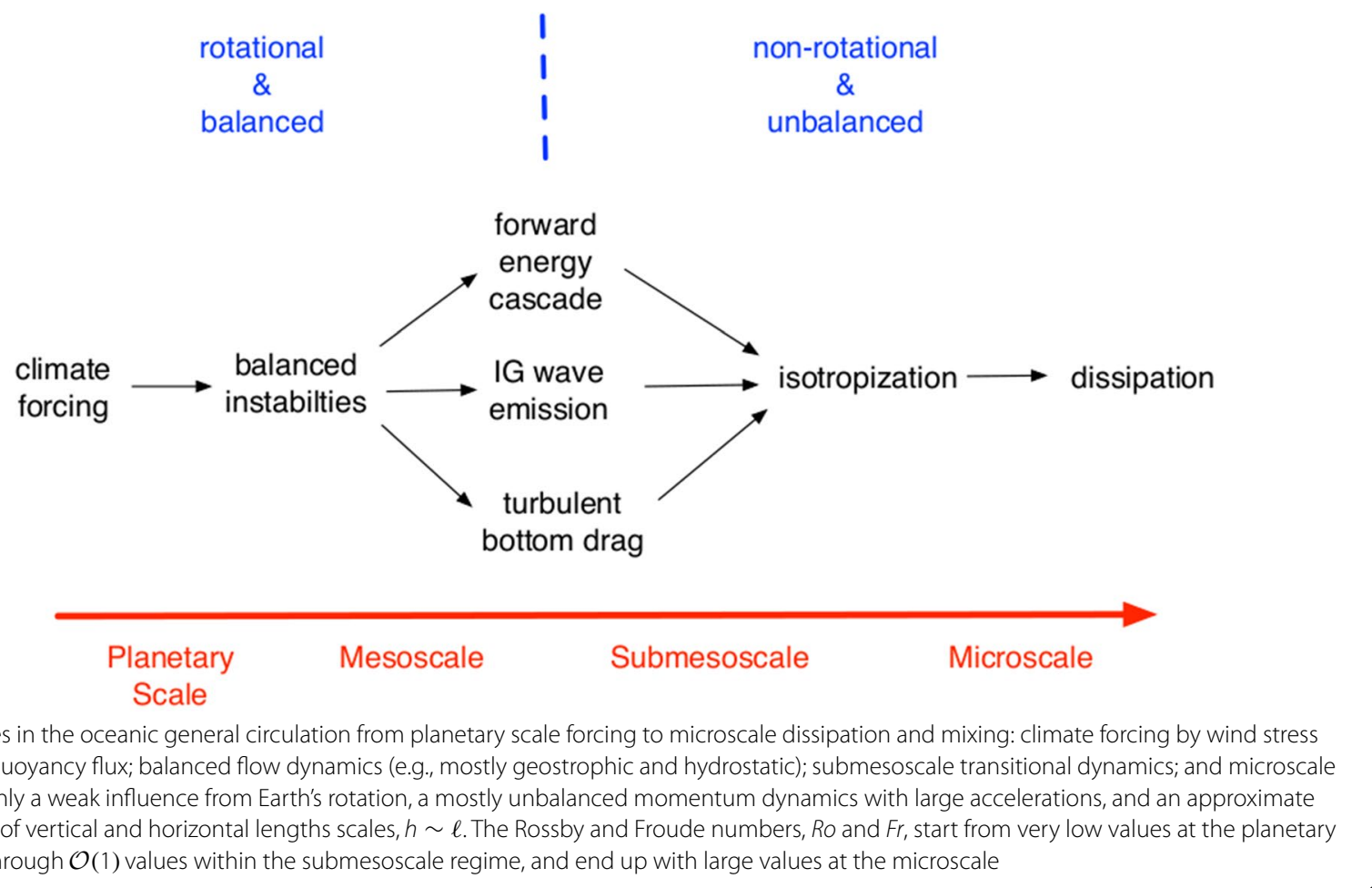

oceanic currents, is mainly due to technical barriers. The SMC space and time scales are awkwardly in between the finer scale sampling from ships, sparse buoys, and floats, and the larger scale sampling from most satellite sensors; their simulation requires large computations that encompass both the mesoscale and submesoscale ranges; and the relevant theories involve difficult nonlinear dynamics. The more recent empowering technologies are high-resolution surface images, multiply-nested computational simulation methods, and, in a few instances, massive swarms of surface drifters (as in the CARTHE experiments in the Gulf of Mexico). As yet there is no widely deployable SMC measurement technique for the subsurface ocean, so simulations are leading the way. Autonomous gliders and ship-towed instruments can provide submesoscale spatial sampling along their tracks, but they are limited to two dimensions and thus often have difficulty in distinguishing SMCs and inertia-gravity waves.

Figure 1 is diagram of the flow of information and energy in the global oceanic circulation. ${ }^{2}$ Their originating source is forcing by surface winds and air-sea

\footnotetext{
${ }^{2}$ The energy and information cycles for the tides and surface gravity waves are largely separate from the general circulation cycle.
}

buoyancy fluxes at the energetic scales of the atmosphere, i.e., mostly on the planetary scale comparable to the size of oceanic basins. The direct oceanic response is oceanic currents on the basin and inter-basin scales, including their narrower transport closures as boundary and equatorial currents. On the other hand, the sink is energy dissipation and information loss that can only be completed at the microscale due to molecular viscosity and conductivity. Currents of different types must connect the source and sink across the intervening scales and dynamical regimes. The first step is geostrophic instabilities of the forced circulation, yielding mesoscale eddies. Through the force-balanced constraints of geostrophic and hydrostatic balance, they are inhibited from further transfers to smaller scales; i.e., they have an inverse cascade of energy (Charney 1971). Leakages out of the mesoscale eddies by partial violation of these force balances continue downscale. Three middle-scale "routes" are depicted in Fig. 1: (1) spontaneous emission of inertiagravity waves from currents, either in the interior or as bottom lee waves, followed ultimately by energy transfer to smaller scales; (2) partly ageostrophic instabilities and forward energy cascade of non-wave (partly balanced) currents; and (3) turbulent bottom drag on currents that generates both bottom boundary-layer turbulence 
and topographic vortical wakes. Below this middle-scale range, three-dimensional turbulence completes the connection to the microscale. While no accurate global accounting of these three routes is yet available, the SMC role in the latter two routes is almost certainly a dominant one. Along the pathway toward the microscale, the character of the currents changes from being highly anisotropic with $h / \ell \ll 1$ and relatively small vertical velocity to approaching isotropy with $h \sim \ell$, as in Kolmogorov's paradigm for universal turbulent behavior at high Reynolds number. In addition, the local $R o$ and $F r$ systematically increase as $h$ and $\ell$ decrease.

The paper is organized by considering the two SMC surface-layer and topographic populations separately in "Lines on the surface" and "Topographic wakes" sections, and it ends with a summary in "Final remarks" section.

This article is adapted from a lecture given at the 2018 annual meeting of the Asia Oceania Geosciences Society (AOGS) in Honolulu, HI. It is intended for a broad audience, and it is more about my experiences and opinions, with some illustrations, rather than about the full evidence and literature behind them. A more extensive and scholarly review article is McWilliams (2016), while this paper is intended to complement it for a more general audience.

\section{Lines on the surface}

SMCs can be difficult to discern in single vertical profiles or time series because of potential confusions with other types of flows. Among the most useful observations are surface images, especially those with high horizontal resolution (subsurface images are rare, but see Fig. 14). By the addition of an extradimension and with dense sampling, patterns emerge, and with experience, they can be interpreted for their underlying phenomena. Images in a horizontal plane (i.e., at the oceanic surface) allow a recognition of sharp convergence lines and small, horizontally recirculating vortices. Several examples are presented in this section.

Besides the recognition of lines of large horizontal density gradients (i.e., fronts), many of these lines exhibit meanders, which is suggestive of frontal instability, likely due to the associated vertical or horizontal shear in the mixed layer. The former is a type of baroclinic instability but with preferred length scales that are in the submesoscale range due to the small surface-layer baroclinic deformation radius (Boccaletti et al. 2007; Fox-Kemper et al. 2008). The latter is a form of classical shear instability that is engendered by the sharpening horizontal gradients caused by active frontogenesis. Both types can be partly ageostrophic because of the large value of the Rossby number.

A snapshot of sea surface temperature (SST) within the California Current System shows both mesoscale eddies and associated submesoscale fronts, filaments, instabilities, and vortices (Fig. 2). A front is defined as a sharp horizontal gradient in density with an extensive central axis in the perpendicular direction (i.e., a line along the surface). A filament is similar, except that it is a narrow horizontal extremum in density. Both light and dense filaments are possible, but dense filaments have much stronger ageostrophic circulation and more rapid frontogenesis, and, hence, are more common in the ocean (McWilliams et al. 2009; Adams et al. 2017). These submesoscale density patterns have associated geostrophic flows along the axis and ageostrophic flows, especially in the cross-axis plane.

A sun-glint reflection pattern in the Mediterranean Sea (Fig. 3) reveals a dense family of lines on the surface. They are due to high concentrations of buoyant surfactants (in this case biogenic scum) that are gathered into narrow lines along the front or filament axes by surface ageostrophic convergence with downwelling jets underneath. The surrounding submesoscale flows give a pattern organization to the lines, thus serving as a means of flow visualization. In this example, at least two scales of SMCs are present. The larger scale of kilometers is a vortex street of a sort which one might plausibly associate with the late stage of an instability of a lateral shear layer. The smaller scale of tens to hundreds of meters is the lines themselves. Within the vortices, the lines have an inwardly spiraling pattern that exposes both cyclonic swirl and a central convergence in the vortex cores. This led Walter Munk to refer to these as "spirals on the sea" (Munk et al. 2000). During the manned space flight era, many examples were photographed by the astronauts. Outside the vortices, the lines indicate a generally larger scale (mesoscale) flow pattern.

A closer view of an SMC convergence line is in Fig. 4, a photograph taken from a ship in the Gulf of Mexico. Here, it is floating seaweed that is gathered along the axis. The cross-line scale is a few tens of meters, and the along-axis scale reaches to the horizon. This example is from a study whose main content was satellite images showing a dense network of such lines spanning a wide region of hundreds of kilometers in the central Gulf of Mexico (Gower et al. 2006).

A more detailed view of a cyclonic spiral vortex comes from a satellite color image showing plankton patterns in the Baltic Sea (Fig. 5). The plankton has high concentrations where gathered into surface convergence lines that are mostly dense filaments. The pattern interpretation is similar to Fig. 3, except that, here, the SMC vortex appears to be somewhat isolated in space. In other such examples, the lines are more abundant and the identifiable vortices are rarer [e.g., in McWilliams (2016)]; this regime is called the "submesoscale soup", perhaps, with 


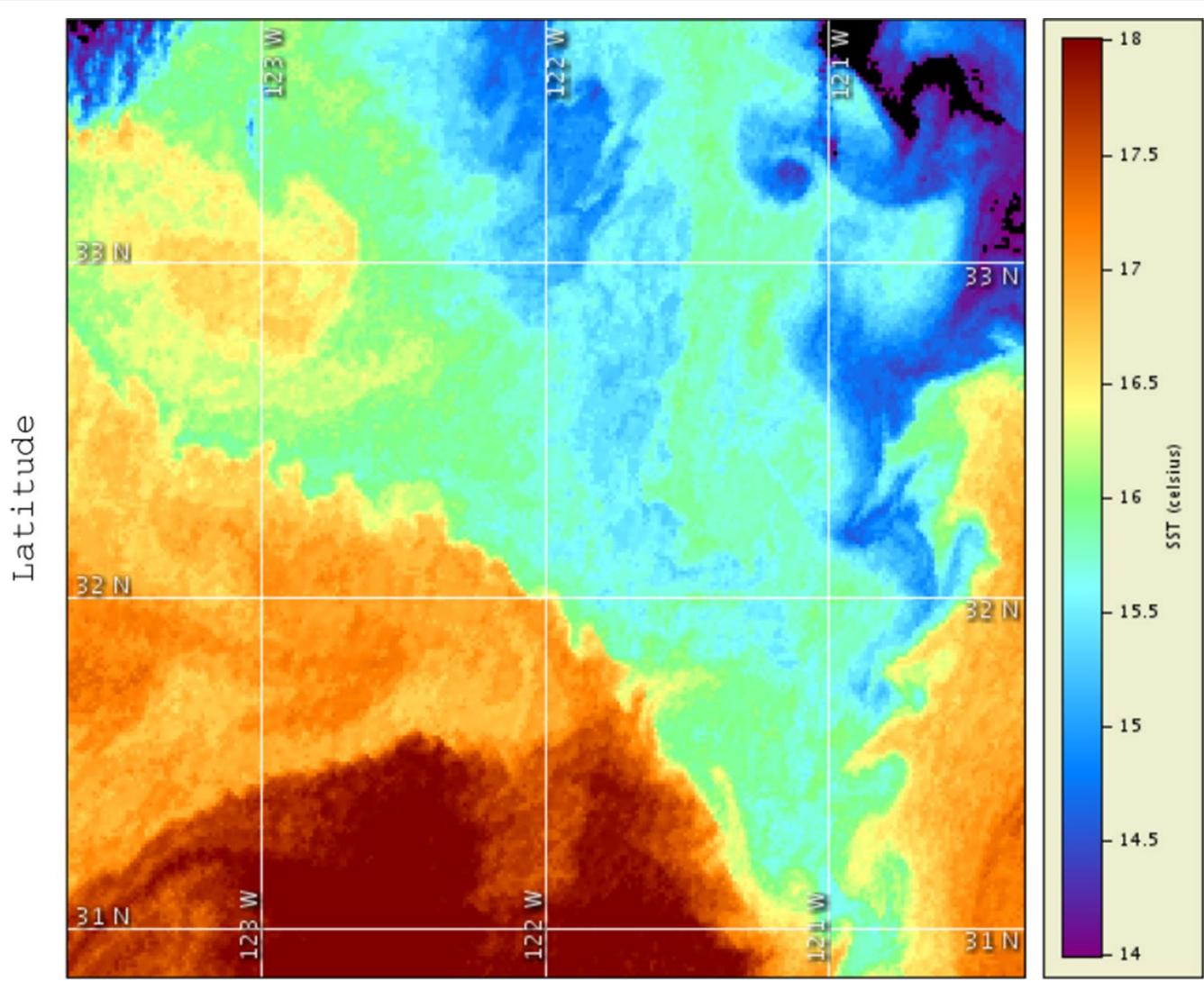

Longitude

Fig. 2 Sea surface temperature of California. Satellite image on June 3, 2006 (NOAA Coastwatch). Notice the mesoscale contrasts over $\approx 200 \mathrm{~km}$ and the submesoscale sharp fronts and filaments on a scale of $\approx 5-10 \mathrm{~km}$. In addition, evident are frontal instabilities (wiggles) and a roll-up into a coherent vortex (in the northeast corner)

vermicelli in mind (e.g., the regions away from the Gulf Stream in Fig. 6).

Such images and photographs are highly informative, but their information is intrinsically subjective. It is quite difficult to get in situ measurements that cover the indicated patterns, though there have been some successes. More generally, however, the quantitative science of SMCs has been advanced by computational simulations. An example for the offshore Gulf Stream is Fig. 6. The experience has been that, in realistic simulations with active mesoscale eddies and associated horizontal density gradients, a sufficiently fine-grid resolution will lead to the spontaneous emergence of SMCs that first arise in the weakly stratified surface layer. The necessary resolution varies with conditions (e.g., region or season), but it is around $d x \approx 1 \mathrm{~km}$; such simulations can be referred to as "submesoscale-permitting", because the full range of submesoscale variability extends down to $\approx 10-100 \mathrm{~m}$, and the latter would have to be reached to be fully "submesoscale-resolving". Nevertheless, simulations show approximately self-similar scaling behavior when $d x$ is varied within this submesoscale range. The associated kinetic-energy horizontal-wavenumber spectra are relatively shallow, $E(k) \sim k^{-\gamma}$, with $\gamma \approx 2$, where $k$ is the horizontal wavenumber. This differs from simulations and altimetric sea-level measurements that show generally steeper spectra (larger $\gamma$ values) in the mesoscale range. The value of the simulation results is mainly in the phenomenological discoveries they have enabled. This is illustrated in Fig. 6 by the variety of different SMC patterns associated with different mesoscale environments. Once the phenomenology is known, then detailed diagnostic analyses and theoretical explanations can be adduced.

One dynamical frontier for submesoscale simulations is the onset of essentially non-hydrostatic behavior. Most SMC simulations to date are made with hydrostatic models. Some estimates for this lower size limit for SMCs are where frontogenesis is arrested by frontal instability and/or where the currents in the forward energy cascade 


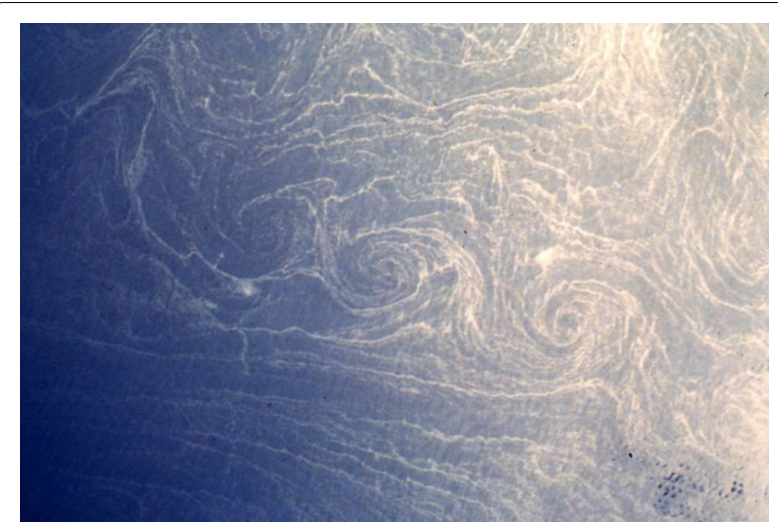

Fig. 3 Spirals on the sea. Sun-glint pattern in a photograph by an astronaut Scully-Power (1986) over the Mediterranean not far off the coast of North Africa. The lines are created by surfactants concentrated in convergence lines that alter the scattered reflection by short-surface gravity waves. Their patterns are organized by submesoscale currents. The vortex diameters are $\approx 5 \mathrm{~km}$, and the surfactant lines are $\approx 100 \mathrm{~m}$ wide. The pattern suggests that a vortex-street roll-up has occurred from a lateral shear instability of some antecedent front, filament, or headland wake

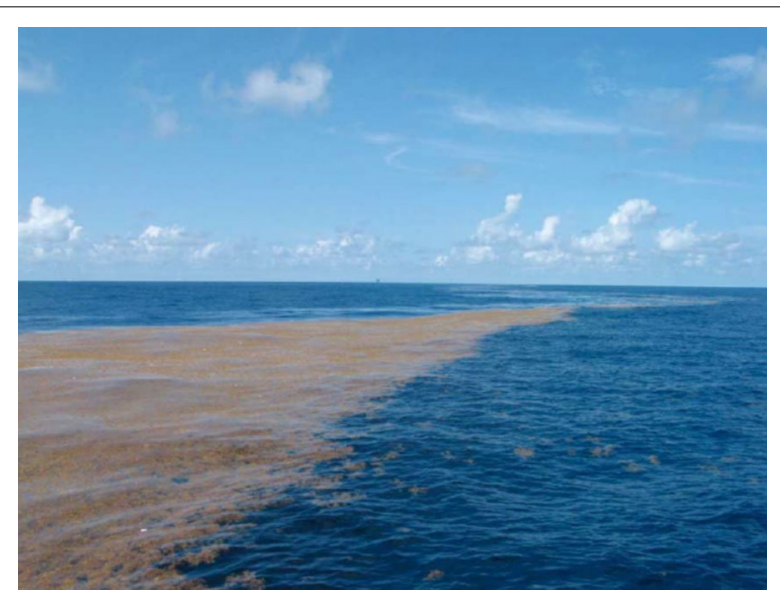

Fig. 4 Surface convergence line. A fantail view of $a \approx 10 \mathrm{~m}$-wide line of buoyant sargassum weed in the Gulf of Mexico concentrated by a submesoscale frontal secondary circulation (Gower et al. 2006). Notice the along-front extent away into the distance, with a suggestion of frontal meanders or instabilities

reach scales where rotation and stratification influences cease to be significant (i.e., relevant $R o$ and $F r$ values are large; Sullivan and McWilliams 2018). Both estimates would yield a horizontal scale in the $10-100 \mathrm{~m}$ range. Whether, in fact, there are important non-hydrostatic effects on SMCs at larger scales remains to be further tested.

In the surface layer, the principal energy source for SMCs is the available potential energy associated with horizontal density gradients on larger scales.

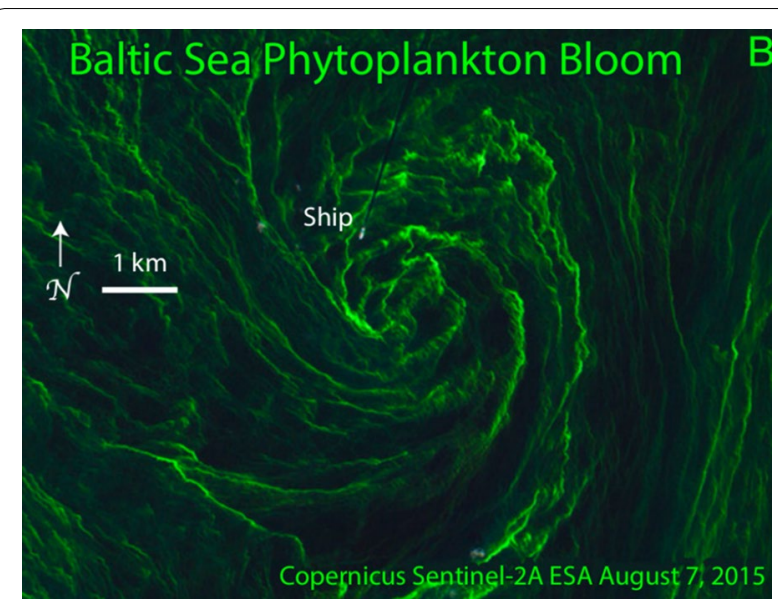

Fig. 5 Submesoscale surface vortex. A satellite image of plankton concentrated in surface convergence lines in the Baltic Sea. The lines indicate a submesoscale central cyclone with spiral arms that are dense filaments. A similar behavior is seen in a set of convergent surface drifter trajectories, first into an arm and then into the cyclone center, in the Gulf of Mexico (D'Asaro et al. 2018), but in a separate event from that depicted in Fig. 4

This energy source can be tapped either by baroclinic instability (often called mixed-layer instability when confined to the weakly stratified surface layer) or by frontogenesis. Frontogenesis is a familiar concept from meteorology because of its frequent manifestation on surface weather maps. The prevailing meteorological interpretation is that fronts are caused by the horizontal strain rate in what is called a deformation flow (e.g., confluence in a horizontal plane) on a larger scale. A sketch of the associated surface frontal structure is in Fig. 7 for both density-front and densefilament configurations. The horizontal buoyancy (i.e., $b=-g \rho / \rho_{0}$, where $g$ is gravity, $\rho$ is density, and $\rho_{0}$ a mean value) gradient will initially sharpen at an exponential rate $\sim \exp [\alpha t]$ as a function of time $t$, if the gradient is favorably aligned in relation to a barotropic deformation flow with a uniform strain rate, no horizontal divergence, and no vorticity; i.e., $\mathbf{u}=\left(u_{d}, v_{d}, 0\right)$ with $u_{d}=-\alpha x / 2$ and $v_{d}=\alpha y / 2$, where $\alpha$ is the horizontal strain rate and $(x, y, z)$ and $(u, v, w)$ are the coordinates and velocity components in the (east, north, and upward) directions (Fig. 7a). Because of this density structure, there is an associated circulation, both along the axis and mostly geostrophic in $v$, and in the cross-axis plane with ageostrophic $u$ and $w$. If the front is uniform along the axis or if an average is taken in this direction (denoted by angle brackets), then the crossaxis velocity is $2 \mathrm{D}$ non-divergent, and it can be represented with a secondary-circulation streamfunction $\Phi$ defined by the following: 


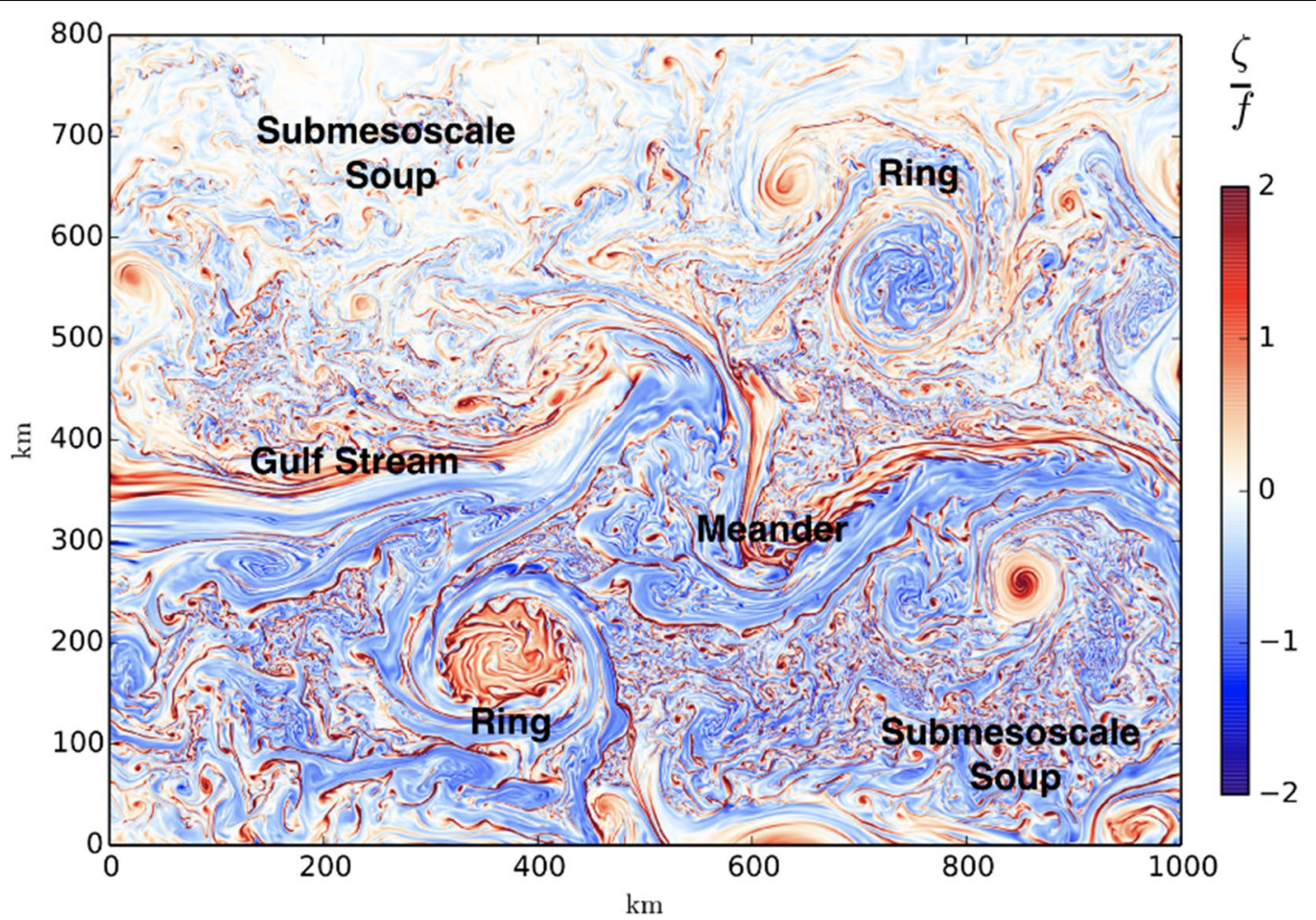

Fig. 6 Simulated offshore Gulf Stream. Vertical vorticity $\left(\zeta=\partial_{x} v-\partial_{y} u\right)$ normalized by $f$ at the surface in the wintertime Gulf Stream after separation from the western boundary in a nested-subdomain simulation (Gula et al. 2015). Notice the meandering Gulf Stream in the center, the northern warm anticyclonic and southern cold cyclonic mesoscale Rings, and the nearly ubiquitous submesoscale features of many different types, including the typical open-sea "soup" away from strong mesoscale currents

$$
(\langle u\rangle,\langle w\rangle)=\left(-\partial_{z}, \partial_{x}\right) \Phi .
$$

For a front, $\Phi$ is a positive monopole indicating a closed circulation loop with upwelling on the light side and surface flow toward the dense side. For a dense filament, $\Phi$ is a dipole, with strong central downwelling. Both patterns imply that $w b>0$, i.e., the conversion of available potential energy to the SMC kinetic energy. Once $\nabla b$ is strong enough, and $\Phi$ is large enough, then the frontogenetic rate increases due to surface horizontal convergence of the secondary circulation, $-\left\langle u_{x}\right\rangle>0$, and it further amplifies until some other process arrests the frontogenesis. The frontal arrest process (discussed at the end of this section) sets the lower size limit for SMCs.

Strain-induced frontogenesis can occur in the ocean, with the principal strain and buoyancy gradients associated with mesoscale eddies or strong currents. More commonly in simulations, however, the dynamical character of the submesoscale fronts is consistent with a combination of a surface density gradient and vertical momentum mixing by boundary-layer turbulence. This situation is called Turbulent Thermal Wind (TTW), which has a linear, steady, surface-layer, incompressible approximation in its horizontal momentum and continuity balances:

$$
\begin{aligned}
& -\partial_{z}\left[v_{v} \partial_{z} u\right]-f v=-\partial_{x} \int^{z} b \mathrm{dz} \\
& -\partial_{z}\left[v_{v} \partial_{z} v\right]+f u=-\partial_{y} \int^{z} b \mathrm{dz} \\
& \partial_{x} u+\partial_{y} v+\partial_{z} w=0,
\end{aligned}
$$

where $v_{v}$ is the vertical eddy viscosity associated with boundary-layer turbulence. Without the buoyancy gradient, this would describe an Ekman layer. Without the turbulent mixing, it would describe a geostrophic current in thermal wind balance. Together, they describe the mixed geostrophic and ageostrophic currents associated with a given $\nabla b$ and $v_{v}$ (McWilliams 2017). The TTW $b$ and $\Phi$ fields for a surface front are shown in Fig. 8. Interestingly, the monopole $\Phi$ pattern is qualitatively the same as for a front in a deformation flow (Fig. 7), and the same similarity occurs for dense filaments. Thus, the TTW circulations are also frontogenetic due to the surface convergence on the dense side or center. The last panel in 

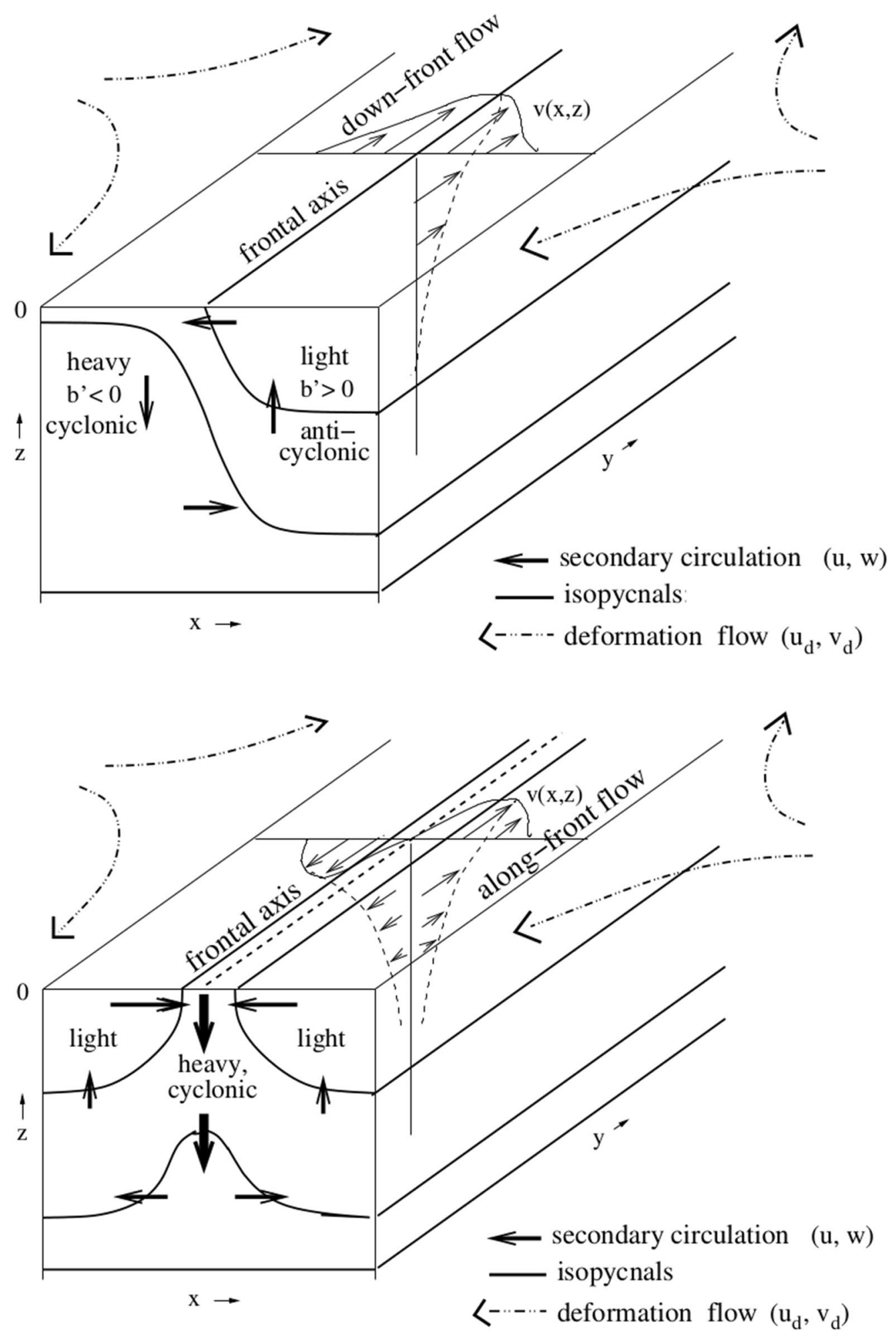

Fig. 7 Frontogenesis by strain. Sketch of surface-layer frontogenesis caused by a larger scale (mesoscale) deformation flow for a front (top) and dense filament (bottom) (McWilliams 2016). There is a geostrophic along-front flow and an ageostrophic secondary circulation in the cross-front plane. With finite Ro, as typical of SMCs, the downwelling and cyclonic vorticity zones are stronger than the upwelling and anticyclonic zones 

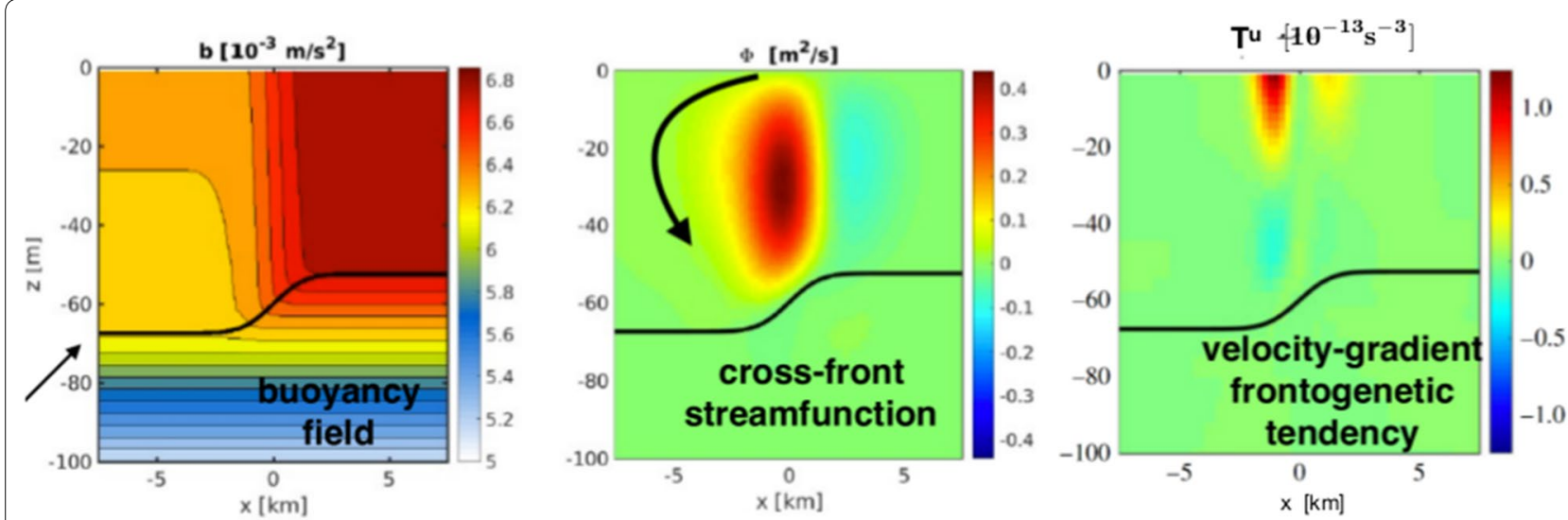

Fig. 8 Frontogenesis by turbulent thermal wind (TTW). The buoyancy field $b$ (left), ageostrophic secondary-circulation streamfunction $\Phi$ (center), and Lagrangian, velocity-gradient frontogenetic tendency, $T^{\mathbf{u}}=D|\nabla \mathbf{u}|^{2} / D t\left[10^{-13} \mathrm{~s}^{-3}\right]$ (right), for an idealized $2 \mathrm{D}$ surface front with vertical mixing (McWilliams 2017). The thick black line, pointed to by the arrow on the left panel, is the boundary-layer depth, and the curved line in the center panel indicates the direction of the secondary circulation. The horizontal convergence on the dense side near the surface (i.e., the upper left region in the buoyancy $b(x, z)$ in left panel induces frontogenesis in both $\nabla b$ and $\nabla \mathbf{u}$

Fig. 8 shows the Lagrangian tendency for the SMC horizontal shear variance, $T^{\mathbf{u}}=D|\nabla \mathbf{u}|^{2} / D t$ : it is strongly positive on the upper dense side of the front. Thus, differential advection by the secondary circulation is the cause of frontogenesis in both the buoyancy gradient and velocity shear.

The simulations discussed above are made with what is called a oceanic circulation model, designed to calculate currents on larger scales, starting from global and

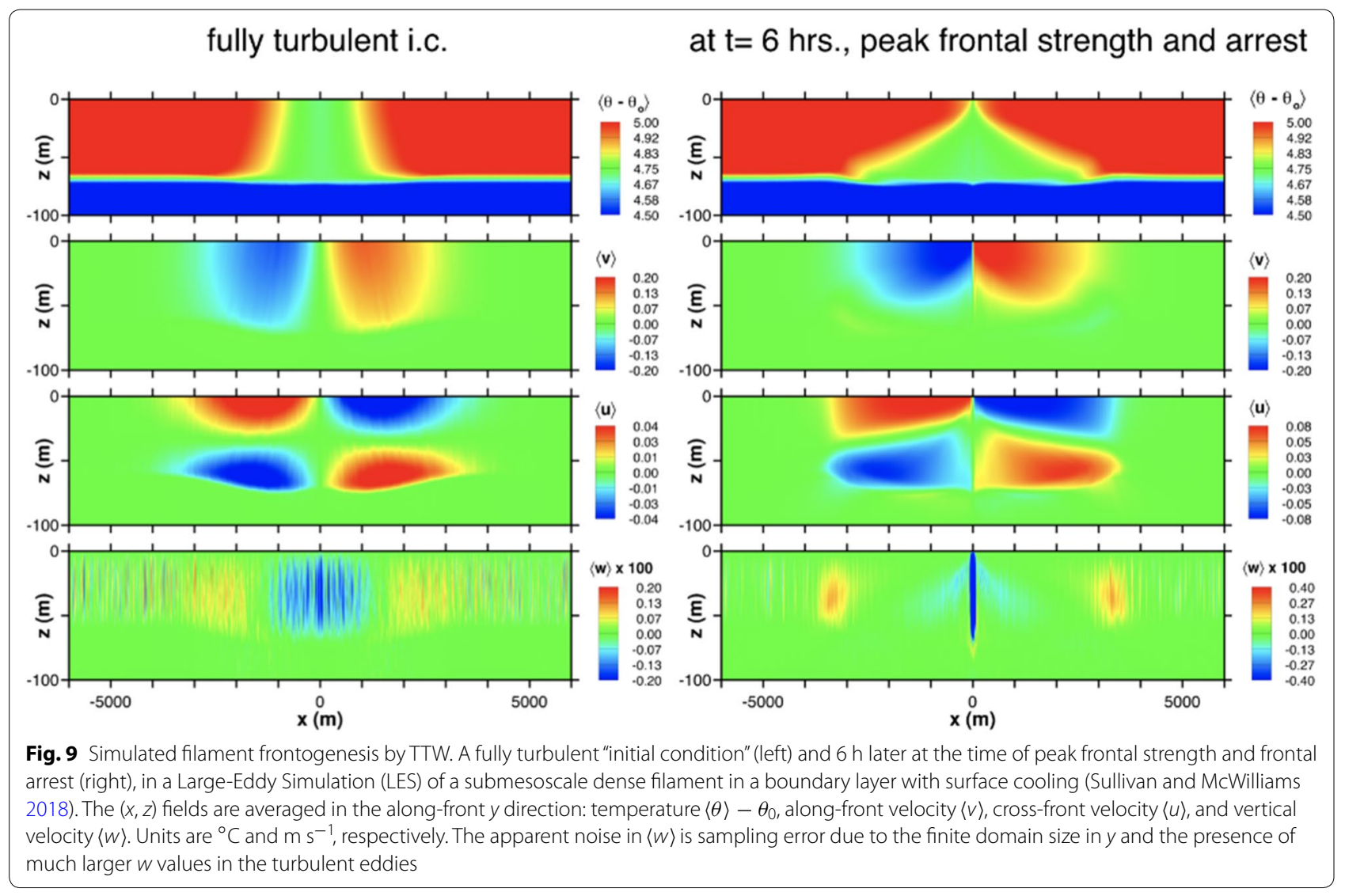



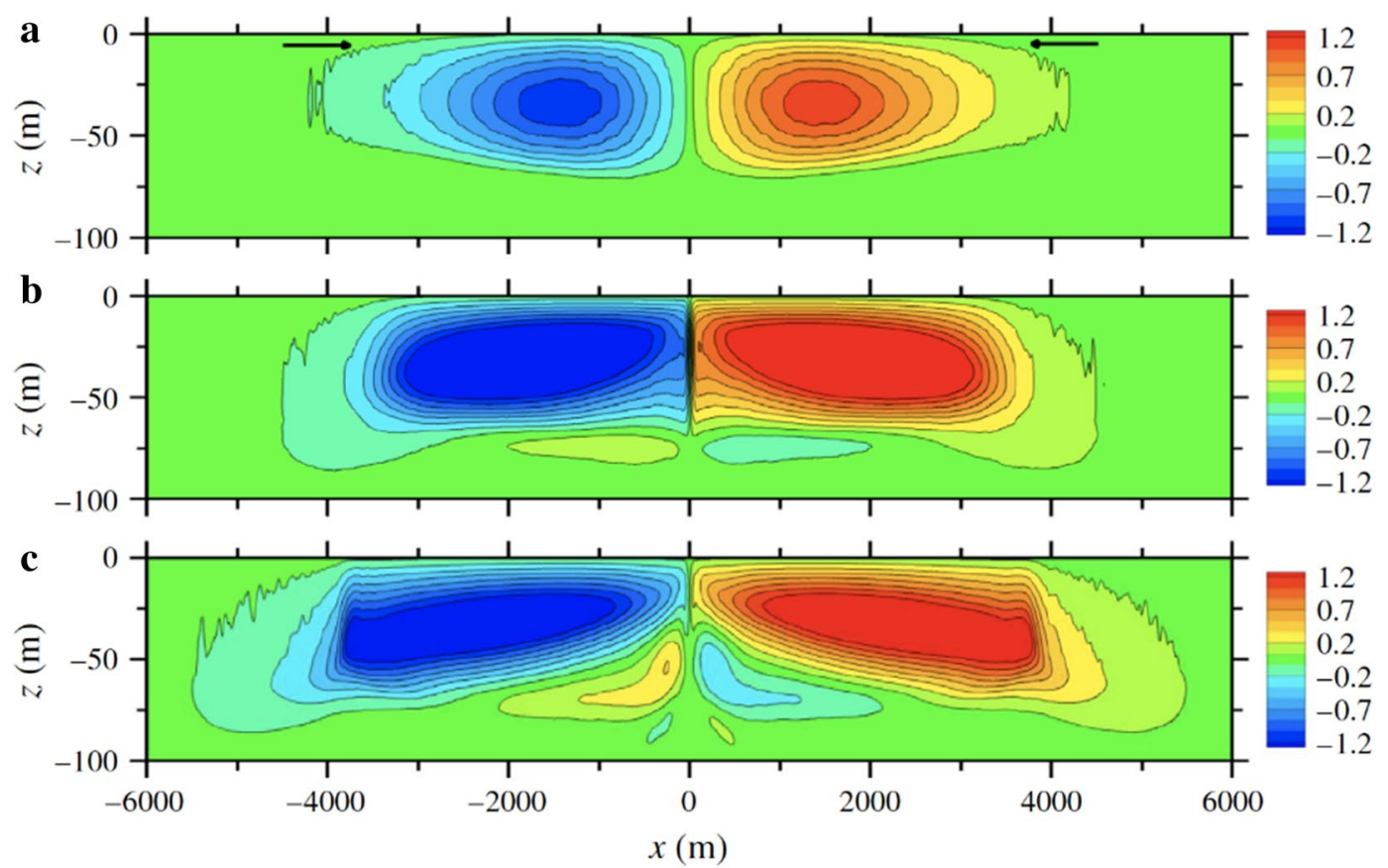

Fig. 10 Filament secondary circulation. The associated cross-front streamfunction $\Phi$ at three different times in the filament life-cycle: (top-to-bottom) initial condition for frontogenesis $(t=0)$, during the peak time of frontal arrest $(t=6 \mathrm{~h}$ ), and during an extensive period of frontal decay ( $t=2$ days). Notice the strong, narrow surface convergence that is the cause of the frontal sharpness. This is for the same simulation as in Fig. 9 (Sullivan and McWilliams 2018)

working downward as computational capacity allows. Most circulation models make the hydrostatic approximation, which seems generally safe to within the submesoscale-permitting regime, apart from whatever high-frequency internal gravity waves might arise. In such a model, small-scale turbulent mixing is parameterized, as with the vertical eddy viscosity $v_{v}$ in (3). Somewhere approaching the dynamical microscale, however, the validity of these simplifications will fail. A dynamical model that is more complete is called a Large-Eddy Simulation (LES), e.g., commonly used for turbulent boundary layers. With respect to SMCs, a large LES calculation that includes both a SMC and its microscale turbulence is a valuable tool for a more fundamental view of their mutual interaction. The following figures are taken for such an LES simulation (Sullivan and McWilliams 2018). It is posed for an isolated dense filament in an otherwise turbulent boundary layer (e.g., due to a surface wind stress and/or convective buoyancy flux). This is a TTW frontogenetic situation. Figure 9 shows the LES initial conditions for the along-axis-averaged temperature (buoyancy) and velocity field in the presence of a fully developed, turbulent, convective boundary layer. Their structure is similar to the filament structure in Fig. 7. From this state, rapid frontogenesis ensues, as seen in the same figure at a time $6 \mathrm{~h}$ later. The filament width has narrowed dramatically at the surface while broadening deeper in the layer. The velocity patterns are similar to their initial shapes, but deformed to have very sharp near-surface gradients at the filament center. In particular, the central $\langle w\rangle<0$ jet has amplified and narrowed substantially. Another view is provided by the cross-axis streamfunction (Fig. 10), whose horizontal convergence in the center similarly narrows and amplifies in accompaniment to the frontogenesis. The time of $6 \mathrm{~h}$ coincides with the peak frontal strength as measured by both the vertical vorticity, $\langle\zeta\rangle=\partial_{x}\langle v\rangle$, and the downward jet, $\langle w\rangle$ (Fig. 11). The duration of the frontogenesis period depends on how strong and wide the filament buoyancy gradient is initially and on the strength of the turbulent momentum mixing that supports the secondary circulation in a TTW momentum balance. This figure also shows that the turbulent kinetic energy (TKE; associated with velocity deviations from the along-front average) also amplifies inside the filament, reaching levels much higher than would occur in a boundary layer without the SMC.

The frontogenesis is arrested by the development of a submesoscale instability of the filament. In this example, the instability is associated with the amplifying 


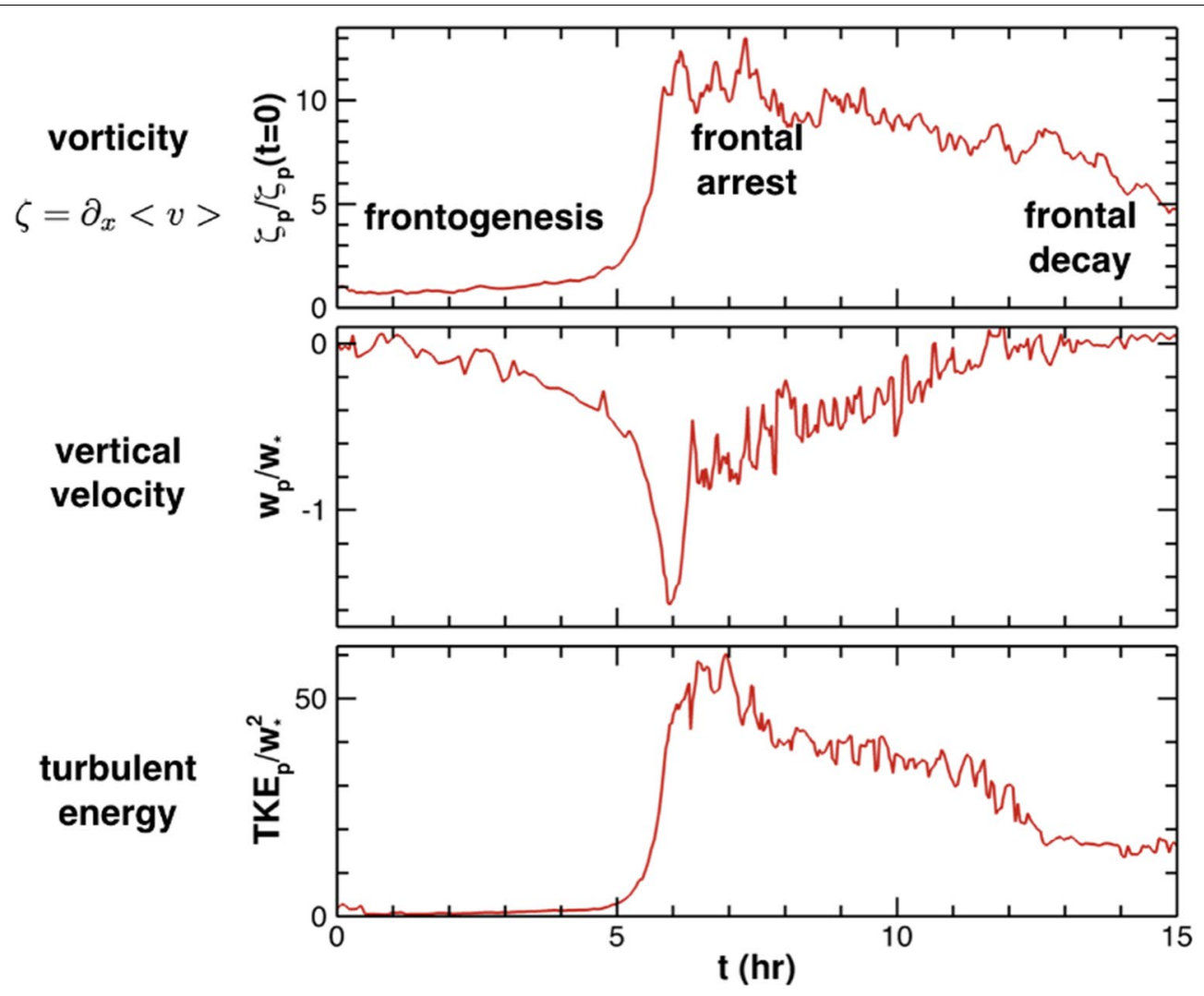

Fig. 11 Filament life-cycle. The associated time series for peak values of the along-front averaged fields in the middle of the filament: peak vorticity normalized by the initial value (top), peak downwelling velocity normalized by the surface cooling scale $w_{*}$ (middle), and peak turbulent kinetic energy normalized by $w_{*}^{2}$. This is for the same simulation as in Fig. 9 (Sullivan and McWilliams 2018)

horizontal shear in $\langle v\rangle$, and evidently, its growth rate exceeds the frontogenetic rate once the filament is narrow enough. As the shear instability amplifies, its eddies have an opposing horizontal Reynolds stress divergence, $-\partial_{x}\left\langle u^{\prime} v^{\prime}\right\rangle$, which counters the advective effect of the frontogenetic secondary circulation. This frontal arrest occurs when the frontal width is $\approx 100 \mathrm{~m}$ for this case, comparable to the boundary-layer depth. In the ocean, fronts are seen with a range of widths from meters to kilometers, so this particular final width is not universal. The SMC circulation and buoyancy structure persist for much longer than the arrest time, with a slow decay in its strength over several ensuing days (Figs. 10, 11). In the filament, after the submesoscale instability has arisen, a turbulent forward energy cascade occurs, illustrating the local pathway from submesoscale to microscale to dissipation (Fig. 1). The associated TKE wavenumber spectrum $E(k)$ (Fig. 12) shows a broad range of variability from the submesoscale peak with its characteristic spectrum slope exponent of $\gamma \approx 2$ into a more fully 3D range with a smaller value of $\gamma \approx 5 / 3$, as expected for boundary-layer turbulence (Thorpe 2005; McWilliams 2016).
Thus, there is an intimate relation between submesoscale currents and boundary-layer turbulence near the surface, with the turbulence providing important mixing effects (e.g., in a TTW evolution) and the former providing important additional TKE excitation and modifying the mixing behavior. This is a research frontier that is almost completely wide open.

\section{Topographic wakes}

Wakes are a familiar fluid dynamical phenomenon: flow past an obstacle generates vorticity in a boundary layer and then separates in the lee; if the Reynolds number ${ }^{3}$ is not small, then the velocity shears within both the boundary layer and the wake are unstable and generate turbulence. The question is how to translate this for the ocean, which involves stratification, rotation, and bottom slopes not side walls. In some instances, the currents are diverted horizontally to approximately follow bathymetric contours without much vorticity generation, and

\footnotetext{
${ }^{3} R e=V L / v$, where $v$ is the molecular viscosity. It is a common parameter indicating how strong momentum advection is compared to momentum diffusion.
} 
in others, the flow is diverted vertically and generates internal gravity lee waves propagating vertically into the interior. However, here, my focus is on instances of significant vorticity generation that lead to unstable wakes, locally enhanced diapycnal mixing and energy dissipation, and formation of submesoscale coherent vortices (SCVs) that are advected into and widely populate the interior ocean (McWilliams 1985).

Because of rotation and stratification, the dynamics of currents is especially sensitive to the vertical vorticity $\zeta=\hat{\mathbf{z}} \cdot \nabla \times \mathbf{u}$, and the Ertel potential vorticity, $q=(f \hat{\mathbf{z}}+\nabla \times \mathbf{u}) \cdot \nabla b$, where the effect of $\zeta$ is emphasized by its multiplication by what is usually the largest component of the buoyancy gradient, $\partial_{z} b$ ( $\hat{\mathbf{z}}$ is the unit vector in the vertical direction). For currents in a boundary layer over a flat bottom, all the vorticity of the turbulence-averaged flow is horizontal. In contrast, currents in a boundary layer along a slope do generate averaged $\zeta$ and $q$ by the geometric argument depicted in Fig. 13: because the bottom boundary layer decreases an interior mean flow to zero at the sloping bottom, there must be an associated horizontal shear (i.e., vertical vorticity, $\zeta^{z}$ ) along a horizontal line extending out into the interior. This is a flow-structure argument, and

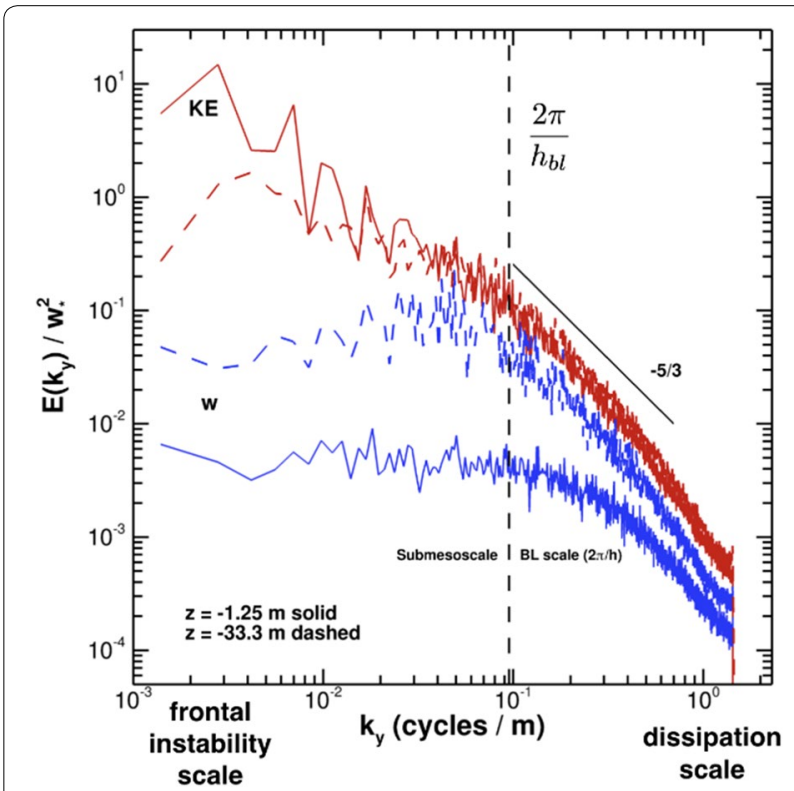

Fig. 12 Filament spectrum during frontal arrest. The associated along-front horizontal-wavenumber $k_{y}$ spectrum of turbulent kinetic energy $E(k)$ and its vertical-velocity $w$ component in the center of the filament at the time of frontal arrest ( $t=6 \mathrm{~h}$; Sullivan and McWilliams 2018). The spectrum peak is associated with a lateral shear instability whose eddy momentum flux, $\left\langle u^{\prime} v^{\prime}\right\rangle$, arrests the frontogenesis by the mean secondary circulation. It is accompanied by a forward cascade of energy to microscale dissipation with a characteristic spectrum slope $\propto k_{y}^{-5 / 3}$. This is for the same simulation as in Fig. 9 (Sullivan and McWilliams 2018) it needs to be extended to encompass the actual rate of $\zeta^{z}$ generation by the along-slope gradients in bottom stress. Nevertheless, the sketch indicates why alongslope near-bottom flows are necessarily a source of vertical vorticity of the flow, and vertical vorticity is a common ingredient in lateral (barotropic) shear instability with small $R o$ and $F r$ values. Furthermore, there is little impetus for currents to separate from a flat-bottom boundary against the gravitational barrier of a stable vertical stratification, whereas it is much more common for currents along a slope to separate while on an intersecting isopycnal surface, whether aided by boundary curvature or even spontaneously.

SCVs are vortices in the interior with limited vertical extent $(\approx 100 \mathrm{~s} \mathrm{~m})$. They typically trap the material concentrations within their cores and often have lifetimes much longer than most individual mesoscale eddies. A particularly famous type is a Meddy (Mediterranean Eddy) SCV, formed from the warm, dense water that flows out of the Mediterranean Sea through the Strait of Gibraltar. The outflow current flows downhill as an entraining density current to a level neutral buoyancy (around $1000 \mathrm{~m}$ depth), turns poleward as a boundary current, separates, and becomes unstable in the interior. An acoustic image of its temperature structure is in Fig. 14. It is an axisymmetric blob of warm water (hence high salinity for its given buoyancy) surrounded by a horizontally recirculating current whose maximum is at its middle depth and is in gradient-wind momentum balance with a central pressure anomaly. A Meddy is a

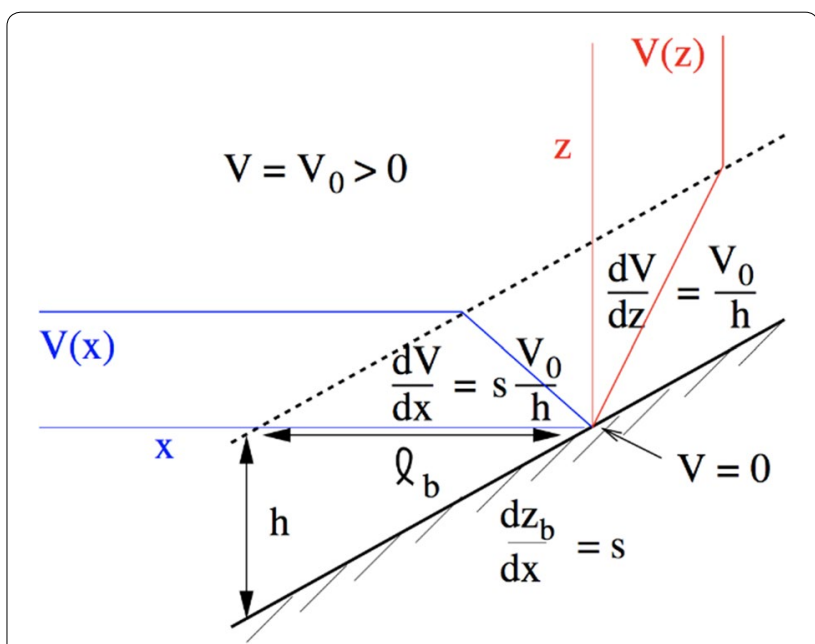

Fig. 13 Drag-induced vorticity generation on a slope. Sketch of vorticity generation in an along-slope current $V(x, z)$ for a uniform interior flow $V_{0}$ and a turbulent bottom boundary layer over a slope with $s=d z_{b} / d x$. The turbulent drag causes the bottom velocity to go to zero, leading to the local vertical profile $V(z)$ (red) with a boundary-layer depth $h$ and horizontal profile $V(x)$ (blue) with vertical vorticity $\zeta=d V / d x$ due to the velocity shear on the horizontal scale $\ell_{b}=h / s$ (Molemaker et al. 2015) 


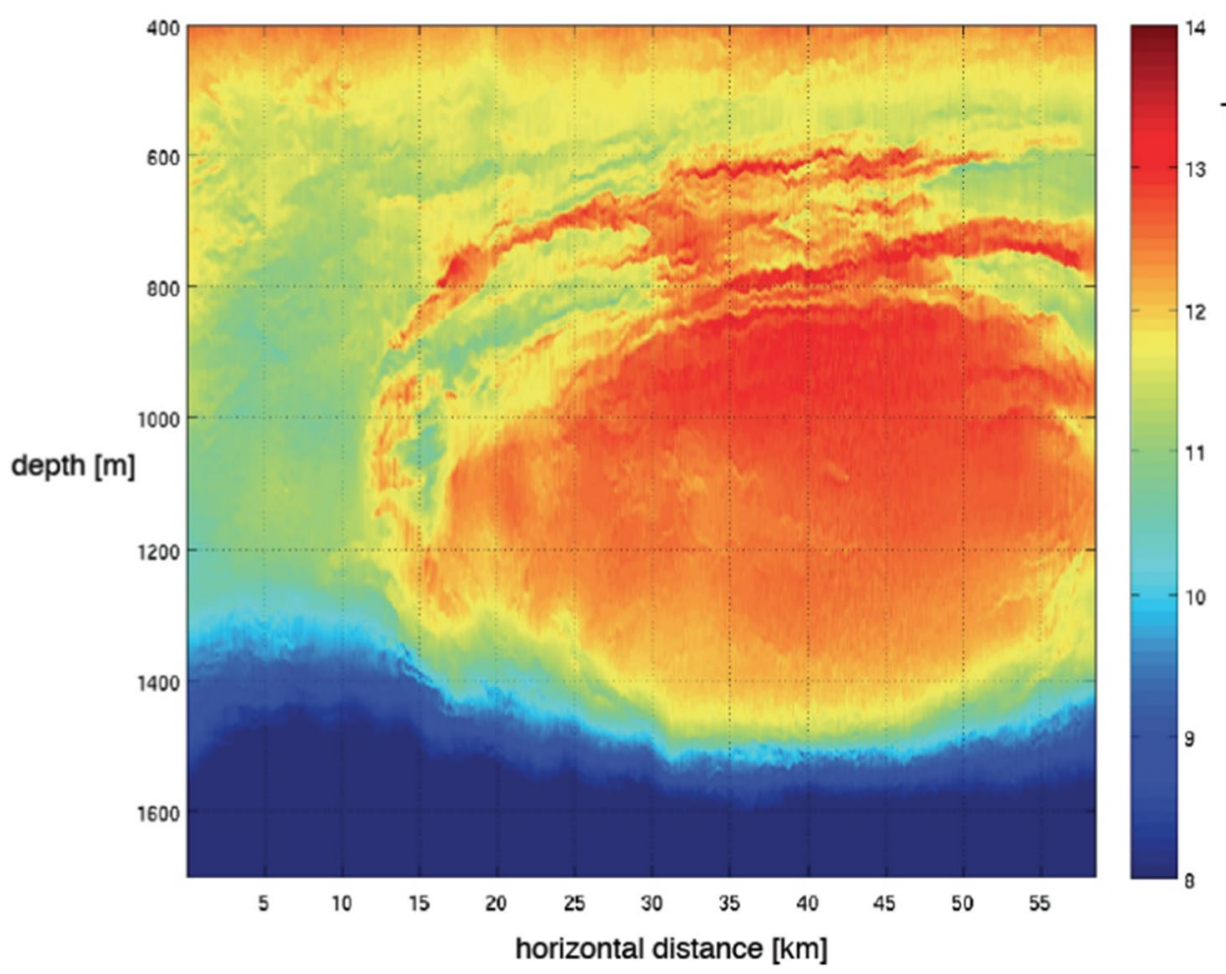

Fig. 14 Meddy SCV. An acoustic tomographic image of a cross section of T in a Mediterranean eddy (Meddy) offshore in the Eastern Atlantic (Papenberg et al. 2010). It is formed by an instability of the separating Iberian slope current fed by the Mediterranean outflow. Lifetimes are up to years, and a few are known to traverse the width of the Atlantic ocean

relatively large type of SCV with a radius of $\approx 20 \mathrm{~km}$ and a half-height of several hundred meters.

A different SCV example is Fig. 15, which shows horizontal float trajectories that recirculate many times around the vortex core with no indication of a significant decay in strength over the two-month sampling period. Separate hydrographic casts made through its core show large chemical property anomalies indicating both material trapping and a lifetime long enough to travel $1000 \mathrm{~km}$ or more (i.e., years).

The hypothesis is that SCVs are generated primarily in topographic wakes where the drag-induced $\zeta$ and $q$ are large enough to make the ensuing vortices strong enough to resist the early disruption by encounters with other, weaker interior currents. Alternatively, a localized vertical mixing event in a stratified region, followed by geostrophic adjustment, can do so, as well (McWilliams 1985; Bosse et al. 2017). Because bottom currents, stable stratification, and topographic slopes are ubiquitous in the ocean at all depths, so SCVs are common, albeit occupying only a small volumetric fraction of the oceanic interior (one estimate for Meddies is $<10 \%$ of the middle depths of the Eastern Subtropical Atlantic).

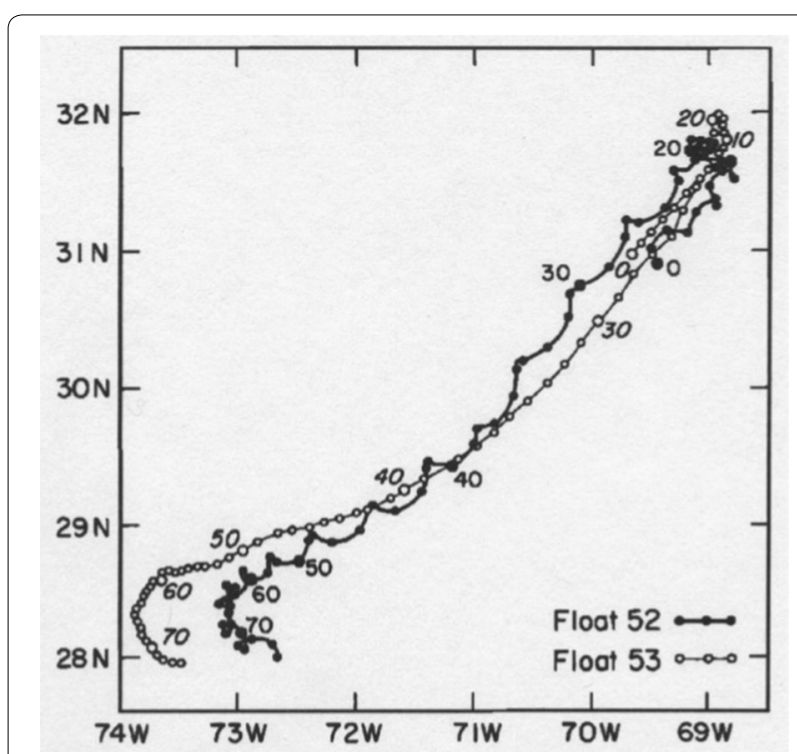

Fig. 15 Float track inside an atlantic SCV. Tracks of two acoustically tracked floats at $700 \mathrm{~m}$ depth near Bermuda (Riser et al. 1986). They were deployed at day 0 about $20 \mathrm{~km}$ apart and stayed close together over a period of more than 70 days while being advected by mesoscale currents. One of these was inside a SCV, the other not. Intersecting hydrographic profiles showed a large, trapped water mass anomaly in $\mathrm{T}-\mathrm{S}$ and $\mathrm{O}_{2}$, indicating a subpolar origin for the SCV 


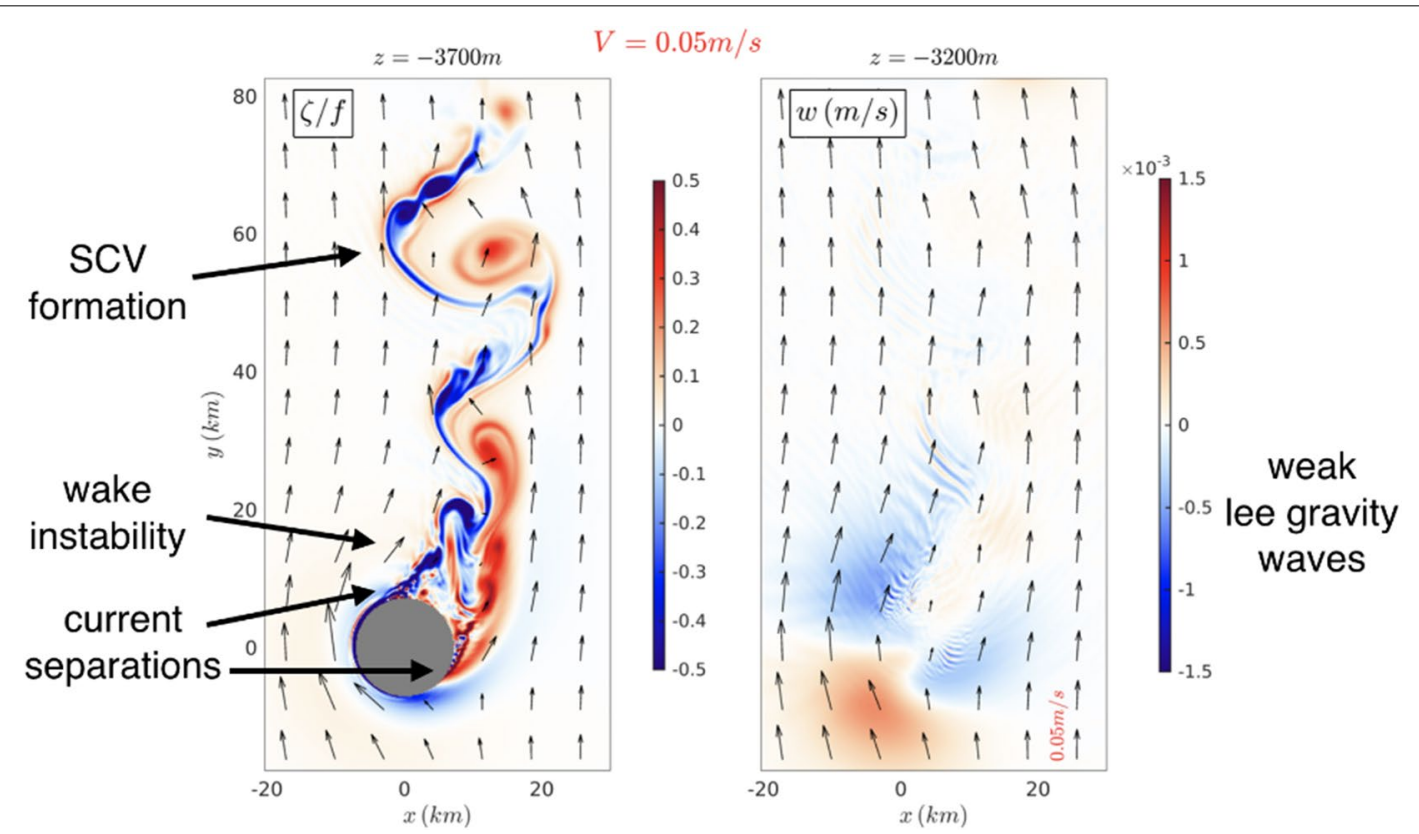

Fig. 16 Seamount wake. Snapshots of normalized vertical vorticity (left) and $w$ (right) in horizontal planes for a simulated flow past a seamount. The upstream flow is steady and uniform, with $V=0.05 \mathrm{~m} / \mathrm{s}$ to the north. The bottom is flat at $z=-4000 \mathrm{~m}$ away from the seamount that has a half-width of $10 \mathrm{~km}$ and a height of $600 \mathrm{~m}$. Vertical vorticity is generated by drag on the slope (Fig. 15), the flow separates into an unstable wake, and the vortex filaments organize into coherent SCVs. In this instance, there is only weak lee gravity wave generation, as shown by the small $w$ above the top of the seamount (Srinivasan et al. 2019)

Model simulations support this hypothesis. In an idealized problem of a uniform, steady inflow past an isolated seamount, SCVs are generated whenever the seamount height is large enough (i.e., the slope is steep enough), the stratification is not weak, and the value of $R o$ is small (Fig. 16). Another example is a realistic simulation of the Subtropical Eastern-Boundary Current System west of North America. There, the poleward California undercurrent flows along the continental slope. It manifests the drag-induced vorticity generation scenario described above. Where it separates, a strong centrifugal instability ${ }^{4}$ arises in the wake (with $f q<0$ ), and submesoscale vortices emerge and mutually interact to form a California Undercurrent SCV (a Cuddy; Fig. 17). Many different Cuddies have been detected off the U.S. West Coast by acoustically tracked recirculating, trapped subsurface float trajectories.

These unstable wakes exhibit strong submesoscale turbulence with a forward energy cascade to dissipation and mixing of material concentrations both along and across

\footnotetext{
${ }^{4}$ Centrifugal (or symmetric or inertial) instability occurs when the Ertel potential vorticity $q$ changes sign within the local domain. Thus, it can only occur when $R o$ or $F r$ is large. In both the surface and topographic SMC populations, it often is triggered by potential vorticity fluxes through the top and bottom boundaries, respectively.
}

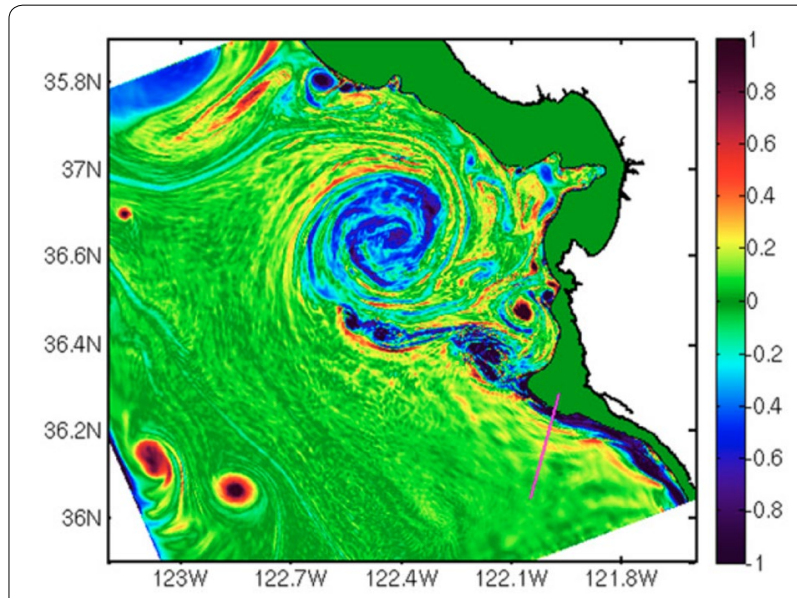

Fig. 17 Cuddy SCV generation by boundary current separation. California undercurrent eddies (Cuddies) form by separation of the California Undercurrent at the headland south of Monterey Bay, CA. This is a simulation snapshot of normalized vertical vorticity, $\zeta / f$, at $150 \mathrm{~m}$ depth. Anticyclonic (blue) vorticity is generated by the bottom drag along the slope and flows north until it separates near Pt. Sur, CA. Centrifugal instability occurs, wake vortices emerge, and then, these smaller vortices merge into a larger Cuddy, seen here in the middle of the Bay at an intermediate stage of self-organization. Subsequently, Cuddies disperse into the interior Pacific by mesoscale currents (Molemaker et al. 2015) 


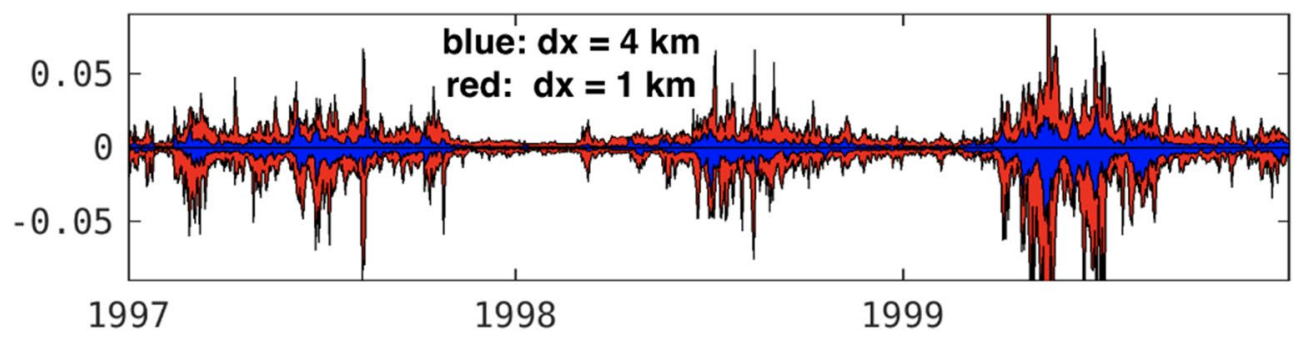

Fig. 18 Submesoscale nutrient flux. A time series of a scatterplot of the product of $w$ and inorganic $N$ for all spatial points at $50 \mathrm{~m}$ depth in the California current in the region $20-200 \mathrm{~km}$ offshore. A submesoscale-permitting simulation ( $d x=1 \mathrm{~km}$; red) shows much greater variability than a mesoscale-resolving one ( $d x=4 \mathrm{~km}$; blue). In this eastern-boundary upwelling system, both simulations have a mean $\overline{w^{\prime} N^{\prime}}<0$, indicating "eddy quenching", i.e., a reduction in primary productivity by burial of unconsumed nutrients by eddy fluxes along the descending isopycnal surfaces. The SMCs enhance $\overline{W^{\prime} N^{\prime}}$ by about $40 \%$ in this comparison (Kessouri et al. 2019).

density surfaces. Thus, this class of topographic SMCs can have widespread influences on the oceanic interior. In my view, it is important to explore these phenomena further, both computationally and observationally.

\section{Final remarks}

This paper focuses on the physical manifestations of SMCs, but there are also biogeochemical and ecological consequences associated with their material fluxes. One illustration is in Fig. 18, showing that the submesoscale nitrogen flux at the base of the euphotic zone acts to limit primary productivity in the eutrophic California Current System. In other more oligotrophic situations, SMCs can enhance productivity by bringing up nutrients from the interior nutricline (Mahadavan 2016). Both instances are due to the relatively large SMC vertical velocity $w$ in the surface layer. More generally, SMCs enhance material exchanges between the turbulent boundary layers and the interior.

In summary, SMCs are active over much of the ocean with large seasonal and geographical variability. They have a distinctive dynamics by being advective, partly ageostrophic, and frontogenetic. There are at least two distinct populations: one associated with surface-layer frontogenesis and the other associated with topographic wakes. Both populations are tightly coupled with the local microscale turbulence, and thus, they are a significant cause of intermittency, heterogeneity, and nonstationary behavior in the surface and bottom boundary layers.

An important open question is how active SMCs are in the ocean interior. Idealized simulations of rotating, stratified turbulence indicate that they should be so, at least in some places where $\mathrm{Ro}$ and $\mathrm{Fr}$ are not too small (Molemaker et al. 2010; Kafiabad and Bartello 2016), e.g., within strong currents and eddies. As yet no realistic oceanic simulations or measurements unambiguously show this to be true, but neither has this issue yet been pushed very hard. Were it to be true, then one would expect a relatively shallow kinetic-energy spectrum, forward energy cascade, and elevated dissipation and diapycnal mixing rates. Of course, SCVs are abundant in the interior, but, by the hypothesis stated in "Topographic wakes" section, these are most likely to have been generated near the topography or in local mixing zones and then moved into the wider ocean.

\section{Authors' contributions}

JM wrote the manuscript. The author read and approved the final manuscript.

\section{Acknowledgements}

This paper was written during a visit to the Kavli Institute for Theoretical Physics, supported in part by the National Science Foundation under Grant No. NSF PHY-1748958. I appreciate continuing research support from the National Science Foundation and the Office of Naval Research. I also appreciate the invitation from AOGS to present a Distinguished Lecture for the Ocean Sciences Section.

\section{Competing interests}

The author declares no competing interests.

\section{Publisher's Note}

Springer Nature remains neutral with regard to jurisdictional claims in published maps and institutional affiliations.

Received: 30 Auqust 2018 Accepted: 14 March 2019

Published online: 25 March 2019

\footnotetext{
References

Adams K, Hosegood P, Taylor J, Sallee JB, Bachman S, Torres R, Tamper M (2017) Frontal circulation and submesoscale variability during the formation of a southern ocean mesoscale eddy. J Phys Ocean 47:1737-1753

Boccaletti G, Ferrari R, Fox-Kemper B (2007) Mixed layer instabilities and restratification. J Phys Ocean 37:2228-2250

Bosse A, Testor P, Mayot N, Prieur L, D'Ortenzio F, Mortier L, Goff HL, Gourcuff C, Coppola L, Lavigne H, Raimbault P (2017) A submesoscale coherent vortex in the Ligurian Sea: from dynamical barriers to biological implications. J Geophys Res Oceans 122:1-22

Charney JG (1971) Geostrophic turbulence. J Atmos Sci 28:1087-1095
} 
D'Asaro E, Shcherbina A, Klymak JM, Molemaker J, Novelli G, Gigand C, Haza A, Haus B, Ryan E, Jacobs GA, Huntley HS, Laxagne HJM, Chen S, Judt F, McWilliams JC, Barkan R, Krwan AD, Poje AC, Ozgokmen TM (2018) Ocean convergence and dispersion of flotsam. PNAS 115:1162-1167

Fox-Kemper B, Ferrari R, Hallberg RW (2008) Parameterization of mixed layer eddies. Part I: theory and diagnosis. J Phys Ocean 38:1145-1165

Gower J, Hu C, Borstad G, King S (2006) Ocean color satellites show extensive lines of floating sargassum in the Gulf of Mexico. IEEE Trans Geosci 44:3619-3625

Gula J, Molemaker MJ, McWilliams JC (2015) Gulf Stream dynamics and fronta eddies along the southeastern U.S. Seaboard. J Phys Ocean 45:690-715

Kafiabad KA, Bartello P (2016) Balance dynamics in rotating stratified turbulence. J Fluid Mech 796:914-949

Kessouri F, McWilliams JC, Bianchi D, Renault L, Deutsch C, Frenzel H (2019) Effects of submesoscale circulation on the nitrogen cycle in the California Current System. Global Biogeochem Cycles (submitted)

Mahadavan A (2016) The impact of submesoscale physics on primary productivity of plankton. Ann Rev Mar Sci 8:161-184

McWilliams JC (1985) Submesoscale, coherent vortices in the ocean. Rev Geophys 23:165-182

McWilliams JC (2016) Submesoscale currents in the ocean. Proc R Soc A 472:20160117-132

McWilliams JC (2017) Submesoscale surface fronts and filaments: secondary circulation, buoyancy flux, and frontogenesis. J Fluid Mech 823:391-432
McWilliams JC, Colas F, Molemaker MJ (2009) Cold filamentary intensification and oceanic surface convergence lines. Geophys Res Lett 36:18602

Molemaker MJ, McWilliams JC, Capet X (2010) Balanced and unbalanced routes to dissipation in an equilibrated Eady flow. J Fluid Mech 654:35-63

Molemaker MJ, McWilliams JC, Dewar WK (2015) Submesoscale instability and generation of mesoscale anticyclones near a separation of the California Undercurrent. J Phys Ocean 45:613-629

Munk W, Armi L, Fischer K, Zachariasen F (2000) Spirals on the sea. Proc R Soc A 456:1217-1280. https://doi.org/10.1098/rspa.2000.0560

Papenberg C, Kaleschen D, Krahmann G, Hobbs RW (2010) Ocean temperature and salinity inverted from combined hydrographic and seismic data. Geophys Res Lett 37:04601

Riser SC, Owens WB, Rossby HT, Ebbesmeyer CC (1986) The structure, dynamics, and origin of a small scale lens of water in the Western North Atlantic thermocline. J Phys Ocean 16:572-590

Scully-Power P (1986) Navy oceanographer shuttle observations, STS 41-G Mission Report. NUSC. Technical Document 7611

Srinivasan K, McWilliams JC, Molemaker MJ, Barkan R (2019) Submesoscale vortical wakes in the lee of a seamount. J Phys Ocean (in press)

Sullivan PP, McWilliams JC (2018) Frontogenesis and frontal arrest for a dense filament in the oceanic surface boundary layer. J Fluid Mech 837:341-380

Thorpe SA (2005) The Turbulent Ocean. Cambridge University Press, Cambridge, p 439

\section{Submit your manuscript to a SpringerOpen ${ }^{\circ}$ journal and benefit from:}

- Convenient online submission

- Rigorous peer review

- Open access: articles freely available online

- High visibility within the field

Retaining the copyright to your article

Submit your next manuscript at $\boldsymbol{\nabla}$ springeropen.com 